\title{
Zwischen Verkündigung und Dialog Intentionen und Inhalte überregionaler christlicher Jugendzeitschriften
}

\author{
von Walter Hömberg und Elke Klimke
}

\section{Jugend, Kirche, christlicher Glaube}

Als „Bruch zwischen Evangelium und Kultur" hat Papst Paul VI. in einer Zeitdiagnose Mitte der siebziger Jahre die von $\mathrm{ihm}$ festgestellte tiefe Entfremdung zwischen den Lebenswelten der Menschen und dem Evangelium bezeichnet. ${ }^{1}$ Diese Entfremdung ist in den vergangenen zwei Jahrzehnten in Deutschland weitergegangen. Die zahlreichen Kirchenaustritte der letzten Zeit sowohl auf katholischer als auch auf evangelischer Seite zeigen dies eindringlich. Darüber hinaus gibt es viele Menschen, die zwar offiziell noch Mitglied in einer Kirche sind, für die jedoch der christliche Glaube offenbar nicht sehr relevant ist.

Insbesondere viele Jugendliche und junge Erwachsene haben sich von der Kirche abgewendet. Hermann Boventer spricht bereits 1987 von einer radikalen Entchristlichung, von der fünf Sechstel der Jugendlichen in Deutschland (West) erfaßt seien. ${ }^{2}$ Wie die Shell-Studie '92 ergeben hat, gehören zwar $90 \%$ aller Jugendlichen in den alten Bundesländern einer Religionsgemeinschaft an; jedoch gehen nur knapp ein Viertel davon häufig zur Kirche, und nur gut $2 \%$ gehören einem kirchlichen Verein an, d.h. können als "Kernmitglied" der Kirche gelten. ${ }^{3}$ Durch die Wiedervereinigung hat sich der Anteil der de facto nicht-christlichen Jugendlichen noch vergrößert: $80 \%$ der ostdeutschen Jugendlichen sind konfessionslos - Glaube und Kirche wurden in der ehemaligen DDR nur geduldet.

Die Abkehr von Glauben und Kirche ist im Zusammenhang mit

Prof. Dr. Walter Hömberg lehrt Kommunikationswissenschaft und Journalistik an der Katholischen Universität Eichstätt. Dipl.-Journ. Elke Klimke arbeitet als Buchhändlerin in Brühl.

1 Arbeitsstelle für Jugendseelsorge der Deutschen Bischofskonferenz/Missio ( $\mathrm{Hg}$.$) , Jugendpastoral und Inkulturation des Glaubens. Postulate für unsere$ Zeit, Düsseldorf 1994, 5.

2 Hermann Boventer, Medien, Religion und Zeitgeist. Anfragen an die christliche und kirchliche Publizistik, in: Communicatio Socialis 20 (1987) H.4, 300-313, hier 304 .

3 Jürgen Eiben, Kirche und Religion - Säkularisierung als sozialistisches Erbe?, in: Jugendwerk der Deutschen Shell ( $\mathrm{Hg}$.), Jugend '92. Lebenslagen, Orientierungen und Entwicklungsperspektiven im vereinigten Deutschland, Bd. 2: Im Spiegel der Wissenschaften, Opladen 1992, 91-104. 
dem Wertewandel zu sehen, der in westlichen Industriegesellschaften seit etwa zwei Jahrzehnten zu beobachten ist. ${ }^{4}$ Der Wertewandel bzw. Wertepluralismus wird auch als Enttraditionalisierung verstanden, d.h. als langfristiger Prozeß zunehmender Erweiterung der individuellen Entscheidungsfreiheiten. ${ }^{5}$ Die größer werdenden Freiräume bedeuten zugleich einen Zwang zu individueller Reflexion, anstatt die von der Tradition vorgegebenen Normen wie selbstverständlich zu befolgen. Individualisierung und Pluralisierung der Gesellschaft gehen Hand in Hand.

Trotz des Individualismus und des Pluralismus der Lebensstile stellt die Jugendforschung übereinstimmend eine Zunahme hedonistisch-materialistischer Orientierungen im zurückliegenden Jahrzehnt fest. Gerade bei den Jüngeren sind die Ansprüche an Konsum, aber auch die Individualitäts- und Privatheitsansprüche stark gewachsen. Darunter wird hier einerseits Originalität verstanden - Selbstverwirklichung, Selbstdarstellung, Abgrenzung -, andererseits Freiheit im Sinne von Entpflichtung, Eskapismus und der Abwehr von gesellschaftlichen, sozialen und beruflichen Zwängen. Die Folge ist ein selbstbewußter Rückzug in die private Idylle. ${ }^{6}$

Daß auch die Religion einen Wandel erfährt, hat Thomas Luckmann bereits vor drei Jahrzehnten diagnostiziert: Religion wird zu einer privaten, individuellen Angelegenheit. Die religiöse Vielfalt wächst sowohl im christlichen Bereich innerhalb und außerhalb der beiden großen Volkskirchen als auch bei Sekten und anderen Gruppierungen; das Individuum kann "nach freiem Belieben aus dem Angebot , letzter" Bedeutungen wählen". ${ }^{7}$ Schon macht das Stichwort „PatchworkReligiosität" die Runde. ${ }^{8}$

4 Vgl. Helmut Klages, Wertedynamik. Über die Wandelbarkeit des Selbstverständlichen, Zürich 1988; Matthias Schuppe, Im Spiegel der Medien: Wertewandel in der Bundesrepublik Deutschland. Eine empirische Analyse anhand von STERN, ZDF MAGAZIN und MONITOR im Zeitraum 1965 bis 1983, Frankfurt am Main/Bern/New York/Paris 1988; Ronald Inglehart, Kultureller Umbruch. Wertwandel in der westlichen Welt, Frankfurt am Main/New York 1989 sowie Heinz Bonfadelli, Jugend und Medien. Befunde zum Freizeitverhalten und zur Mediennutzung der 12- bis 29jährigen in der Bundesrepublik Deutschland, in: Media Perspektiven 1986, H.1, 1-21, hier 5.

5 Vgl. Gertraud Nunner-Winkler, Veränderte Wertorientierungen, neue Identitätskonzepte, in: Leiterkreis der Evangelischen Akademien in Deutschland (Hg.), Zukunftsforum Jugend 2000. Informationsdienst Nr. 3, Frankfurt am Main 1990, 3-8, hier 4.

6 Vgl. Heiner Barz, Religion ohne Institution? Eine Bilanz der sozialwissenschaftlichen Jugendforschung. Jugend und Religion, Bd. 1, Opladen 1992, 29.

7 Thomas Luckmann, Die unsichtbare Religion, 2. Aufl. Frankfurt am Main 1993, 141.

8 Georg Schmid, Vorwort, in: Barz, Religion ohne Institution?, a.a.O., 9f., hier 9. 
Die meisten Jugendlichen beschäftigen sich zwar nach wie vor mit dem christlichen Glauben; ein großer Teil verfügt jedoch über kein ausgeprägtes christliches Selbstverständnis. ${ }^{9}$ Bei ihrer Auseinandersetzung mit Religion scheint für die Jugendlichen die Frage nach der Existenz Gottes ein zentrales Thema zu sein. Es wird kaum bezweifelt, $\mathrm{da}$ es einen vom Menschen unabhängigen Gott gibt. Das traditionelle christliche Gottesbild spielt dabei allerdings keine große Rolle mehr. ${ }^{10}$ Auch Jesus wird nach übereinstimmenden Ergebnissen verschiedener Untersuchungen von den Jugendlichen kaum mehr als Mittler-Instanz zwischen Gott und den Menschen gesehen. Ebenfalls kaum noch Bedeutung hat die christlich begründete Hoffnung auf Auferstehung ${ }^{11}$, obwohl sich der Glaube an ein Weiterleben nach dem Tod wieder stärker ausbreitet: Glaubte 1984 gerade jeder zweite Jugendliche daran, so waren es 1991 schon $58 \% .12$

Darüber hinaus koppeln sich die Sinnfragen immer stärker von einem christlichen Hintergrund ab: Daß das Leben überhaupt Sinn hat, darüber sind sich die 18- bis 29jährigen mit älteren Befragten einig; jedoch müsse man ihm - so die meistgenannte Antwort - selbst einen Sinn geben. ${ }^{13}$ Andererseits wird auch bei Jugendlichen ein großes Interesse an neuen religiösen Strömungen registriert, das auf ein durchaus vorhandenes Bedürfnis nach Religiosität und Spiritualität hinweist. Bei einem Teil der Jugendlichen ließen sich Neigungen zu okkulten Praktiken feststellen: Ein Viertel der von Hartmut Zinser befragten Berliner Schüler hat nach eigenen Angaben mit Pendeln, Kartenlegen und Gläserrücken Erfahrungen gemacht. ${ }^{14}$ Dagegen ist das Interesse der 14- bis 19jährigen am "Psycho-Markt" von Esoterik und New Age nicht sonderlich groß - ganz im Gegensatz zu den Erwachsenen. ${ }^{15}$

9 Vgl. Waltraud Sziegaud-Roos, Religiöse Vorstellungen von Jugendlichen, in: Arthur Fischer' (Hg.), Jugendliche und Erwachsene '85. Generationen im Vergleich, Bd. 4: Jugend in Selbstbildern, Opladen, 1985, 334-386.

$10 \mathrm{Vgl}$. Karl Ernst Nipkow, Bildung als Lebensbegleitung und Erneuerung. Kirchliche Verantwortung in Gemeinde, Schule und Gesellschaft, Gütersloh 1990, 359-397 sowie Andreas Feige, Erfahrungen mit Kirche. Daten und Analysen einer empirischen Untersuchung über Beziehungen und Einstellungen Junger Erwachsener zur Kirche, 2. Aufl. Hannover 1982, 3-179.

11 Vgl. Karl Ernst Nipkow, Erwachsenwerden ohne Gott? Gotteserfahrung im Lebenslauf, München 1987, 64f.

12 Eiben, Kirche und Religion - Säkularisierung als sozialistisches Erbe?, a.a.O., $102 f$.

13 Vgl. Ingrid Lukatis/Wolfgang Lukatis, Jugend und Religion in der Bundesrepublik Deutschland, in: Ulrich Nembach ( $\mathrm{Hg}$. ), Jugend und Religion in Europa. Bericht eines Symposions, 2. Aufl. Frankfurt am Main/Bern/New York/Paris 1990, 107-144, hier 116f., $139 f$.

14 Hartmut Zinser, Okkultismus unter Berliner Schülern, in: Materialdienst der EZW 1990, Nr. 10, 273-290, hier 276.

$15 \mathrm{Vgl}$. Gerhard Schmidtchen, Sekten und Psychokultur. Reichweite und Attraktivität von Jugendreligionen in der Bundesrepublik Deutschland, Freiburg/Basel/Wien 1987, 121. 
Immer weniger Jugendliche finden den Weg in die Kirchen. Auf die Multi-Media-Generation der neunziger Jahre wirken die "salbungsvolle" Sprache des Pfarrers und Kirchenlieder aus dem 16. Jahrhundert oft antiquiert und überholt. Die christlichen Inhalte bleiben auf der Strecke. Das haben auch die Kirchen und ihre Einrichtungen erkannt, und deshalb versuchen sie verstärkt, die jungen Leute auf jugendgemäBere Art zu erreichen. Da Jugendliche besonders gut auf Medien ansprechen ${ }^{16}$, spielen christliche Jugendzeitschriften, die die Sprache der Heranwachsenden sprechen, in diesem Zusammenhang eine wichtige Rolle.

\section{Anlage und Durchführung der Untersuchung}

Unser Forschungsinteresse richtete sich auf die Frage, welche Intentionen die überregionalen christlichen Jugendzeitschriften verfolgen, die in der Bundesrepublik Deutschland erscheinen und sich an ein allgemeines Publikum richten, und wie sie diese konkret umsetzen. Unter Berücksichtigung der aufgestellten Kriterien - überregional verbreitete christliche Jugendzeitschriften für alle Jugendlichen ab zwölf Jahren ${ }^{17}$ wurden bundesweit insgesamt zwölf Titel ermittelt; es handelt sich um eine Vollerhebung. Im einzelnen sind dies sechs katholische Zeitschriften (,Junge Zeit", "Kontraste", "Provo", "Komm mit", "17", "Aktion“), fünf evangelische Blätter ("Die Glocke“, „Baustein“, "Contrapunkt”, "Dran", „Exact”) und ein freikirchliches Jugendmagazin ("Champ").

Um Informationen über die Konzeptionen, Intentionen, Zukunftsperspektiven und Zielgruppen sowie über die wirtschaftliche und personelle Situation der zwölf christlichen Jugendzeitschriften zu

16 Vgl. z.B. Heinz Bonfadelli u.a.: Jugend und Medien. Eine Studie der ARD/ZDF-Medienkommission und der Bertelsmann-Stiftung. Frankfurt am Main 1986 sowie Birgit van Eimeren/Walter Klingler: Elektronische Medien im Tagesablauf von Jugendlichen. Nutzungsdaten 14- bis 19jähriger zu Fernsehen, Video, Hörfunk und Tonträgern, in: Media Perspektiven 1995, H. 5, S. 210-219.

Die Altersgrenzen mußten selbst festgelegt werden, da es in der einschlägigen Literatur keine allgemein anerkannten Abgrenzungen für Beginn und Ende der Jugendphase gibt. Für die Auswahl der Untersuchungsobjekte wurde jeweils auf die Altersangaben der Herausgeber zurückgegriffen. In Anlehnung an die Studie von Manfred Knoche/Monika Lindgens/Michael Meissner (Jugendpresse in der Bundesrepublik Deutschland, Berlin 1979) haben wir die Abgrenzung zur Kinderzeitschrift bei zwölf Jahren gezogen. Diese Entscheidung erscheint vor dem Hintergrund gerechtfertigt, daß in diesem Alter die Geschlechtsreife einsetzt und damit ein mehr oder weniger "objektives" Kriterium für den Beginn der Jugendphase vorhanden ist. Auf die genaue Festlegung einer oberen Altersgrenze wurde verzichtet; vielmehr richtete sich die Auswahl danach, ob sich die Zeitschriften selbst als Jugendzeitschrift sehen. 
bekommen, wurde im Sommer 1994 unter den Chefredakteuren eine schriftliche Befragung durchgeführt. ${ }^{18}$ Die Chefredaktion von "Bravo" das auflagenstärkste Jugendmagazin wurde als Vergleichsmedium stellvertretend für die kommerzielle Jugendpresse mituntersucht erhielt einen leicht veränderten Fragebogen, aus dem die spezifisch christlichen Aspekte herausgenommen worden waren.

Der Fragebogen an die christlichen Jugendzeitschriften umfaßte sechs Seiten mit insgesamt 24 Fragen, davon $15 \mathrm{mit}$ verschiedenen Antwortvorgaben. Der Bogen an „Bravo“ enthielt ebenfalls 24 Fragen, wovon bei 13 Antwortmöglichkeiten vorgegeben waren. Weil nicht davon ausgegangen werden konnte, daß den Befragten viel Zeit für das Ausfüllen des Fragebogens zur Verfügung stehen würde, und aus Gründen der besseren Vergleichbarkeit überwiegen die geschlossenen Fragen. Häufig folgten jedoch offene Fragen zum selben Aspekt; so hatten die Befragten die Möglichkeit, zusätzlich weitere ihnen wichtige Punkte anzusprechen.

Die Umsetzung der genannten Intentionen und Konzeptionen im Heft wurde exemplarisch an drei der zwölf Jugendzeitschriften überprüft. Kriterien für die Auswahl waren Magazincharakter, unterschiedlicher Konfessionshintergrund (evangelisch, katholisch, freikirchlich) und hohe Auflage. Die Wahl fiel auf die "Junge Zeit", die unter den katholischen Zeitschriften die zweithöchste Auflage hat (44.000 verkaufte Exemplare) ${ }^{19}$, die "Glocke“, die mit einer Verkaufsauflage von 26.000 Exemplaren das auflagenstärkste unter den evangelischen Blättern ist, und auf das einzige freikirchliche Magazin "Champ" mit einer Auflage von 11.000 verkauften Exemplaren.

Mit Hilfe der Inhaltsanalyse haben wir die inhaltliche und formale Gestaltung der drei ausgewählten Zeitschriften systematisch erfaßt. Dazu wurden pro Zeitschrift vier Ausgaben untersucht - eine aus jedem Quartal des Jahres 1993, und zwar aus den Monaten März, Juni, September und Dezember. Auch hier wurde „Bravo“ als kommerzielles Jugendmagazin in die Untersuchung einbezogen. Ziel war es, das Themenspektrum, das äußere Erscheinungsbild, die Sprache und die Art der Aufbereitung zu vergleichen. Im Zentrum der Untersuchung standen dabei die Inhalte. Grundvoraussetzung für eine solche Erhebung ist systematisch, d.h. nach genau festgelegten Kriterien erhobenes Datenmaterial. Diese Voraussetzung wird von der Inhaltsanalyse erfüllt,

18 Vgl. u.a. Jürgen Friedrichs, Methoden empirischer Sozialforschung, 14. Auff. Opladen 1990, 236-246; Liselotte Wilk, Die postalische Befragung, in: Kurt Holm (Hg.), Die Befragung 1. Fragebogen - Die Stichprobe, München 1975, 187-200.

19 Nur die katholische Zeitschrift "Aktion" hat mit 120.000 Exemplaren eine höhere Auflage. Aufgrund der Themengebundenheit war dieses Blatt jedoch nicht für die Inhaltsanalyse geeignet. Der Vergleichbarkeit wegen wurden Zeitschriften mit Magazincharakter ausgesucht. 
bei der es sich um eine „empirische Methode zur systematischen, intersubjektiv nachvollziehbaren Beschreibung inhaltlicher und formaler Merkmale von Mitteilungen" handelt. ${ }^{20}$ Die vorliegende Studie beschränkte sich auf eine Frequenzanalyse, bei der Häufigkeiten bestimmter Merkmale ausgezählt wurden.

Strenggenommen wurden zwei einzelne Inhaltsanalysen mit jeweils unterschiedlichen Erhebungseinheiten durchgeführt: Bei der ersten Analyse bildete jede Heftausgabe eine Analyseeinheit, wobei das äußere Erscheinungsbild anhand von sechs Kategorien wie Umfang, Farbanteil, Bild-/Textverhältnis und Anzeigenanteil überprüft wurde. Bei der zweiten, umfangreicheren Analyse bildeten die einzelnen Artikel die Erhebungseinheiten. Als Artikel definiert wurde ein in sich abgeschlossener, auf ein bestimmtes Thema bezogener redaktioneller Beitrag (verbaler als auch non-verbaler Zeichenträger, d.h. Text oder Bild/Symbol), den eine Überschrift, ausgesparte Zeilen und/oder fett gesetzte Anfangsbuchstaben oder -worte von den anderen Beiträgen trennen. ${ }^{21}$

Die einzelnen Artikel wurden anhand von insgesamt 28 Kategorien überprüft. Dies waren unter anderem Länge, Darstellungsart, Anzahl der Illustrationen und Schriftarten, .Zugehörigkeit zu einer Themeneinheit, Darstellungsform, Sprachstil, Äußerungen Jugendlicher oder Stellungnahmen kirchlicher Amtsinhaber. Des weiteren haben wir die Themen analysiert, wobei jeder Artikel einem Hauptthema zugeordnet wurde. Thematische Kategorien wie Kultur, Sport, Individueller Lebensbereich und Gesellschaftsrelevante Themen wurden auf einen eventuell vorhandenen christlichen Bezug sowie auf dessen Art und Ausprägung hin untersucht. 22 Für jede Untersuchungseinheit wurde ein Codierbogen ausgefüllt, so daß nach Beendigung des Codiervorganges für die Analyse der äußeren Erscheinungsbildes 12 Bögen und für die Analyse der einzelnen Artikel insgesamt 1.730 Codierbögen vorlagen.

Nach Auswertung der schriftlichen Befragung und der Inhaltsanalyse wurden mit den Chefredakteuren Leitfadengespräche durchgeführt. ${ }^{23}$ Hierbei bot sich die Gelegenheit, die Befragten mit Ergebnissen

20 Werner Früh, Inhaltsanalyse. Theorie und Praxis, 3. Aufl. München 1991, 24.

21 Vgl. Jürgen Hüther/Hildegard Scholand/Norbert Schwarte, Inhalt und Struktur regionaler Großzeitungen, Düsseldorf 1973, 35.

22 Handelte es sich bei den Artikeln um Such-/Kontaktanzeigen, Inhaltsverzeichnis, Witz/Cartoon, Rätsel/Quiz, Fernsehprogramm/Hitliste oder Impressum/Vorschau, wurden aufgrund ihres für die vorliegende Untersuchung irrelevanten Inhalts nur die Kategorien Länge und Darstellungsart erfaßt. Das betraf 535 Fälle.

23 Vgl. Helmut Kromrey, Empirische Sozialforschung. Modelle und Methoden der Datenerhebung und Datenauswertung, 3. Aufl. Opladen 1986, 196-215, hier 211. - Die Gesprächstermine lagen zwischen dem 28. September und 14. Oktober 1994. In einem Fall war der Chef vom Dienst Gesprächspartner. 
zu konfrontieren und sie zu Widersprüchen zwischen Angaben im Fragebogen - beispielsweise hinsichtlich der Konzeption - und der tatsächlichen Umsetzung im Heft, wie sie die Inhaltsanalyse ergeben hatte, Stellung nehmen zu lassen. Es ging dabei vor allem um Einordnung und Kommentierung, aber auch um die Unterschiede zu kommerziellen Jugendzeitschriften, insbesondere zu „Bravo".

Mit Hilfe der drei genannten Methoden Inhaltsanalyse, Befragung und Leitfadengespräch sollten folgende Hypothesen überprüft werden:

- Die Chefredakteure der zwölf überregionalen christlichen Jugendzeitschriften verfolgen eine christliche Intention, die sich in den publizistischen Konzeptionen durch die Betonung der Wichtigkeit von christlichen Inhalten niederschlägt. (H. 1)

- Die Chefredakteure der zwölf überregionalen christlichen Jugendzeitschriften halten es für wichtig, sich an den Interessen der Jugendlichen $\mathrm{zu}$ orientieren. (H. 2)

- Mit Blick auf die konsumorientierte und schnellebige Jugend, auch was die Mediennutzung betrifft, sind die Artikel der drei näher untersuchten christlichen Jugendzeitschriften überwiegend kurz gehalten. Auch bei "Bravo" sind die Beiträge vorwiegend kürzer als eine Seite. (H. 3)

- Die drei näher untersuchten christlichen Jugendzeitschriften orientieren sich sprachlich an den Jugendlichen, indem sie sich - vergleichbar zu "Bravo" - um einen jugendlichen Sprachstil bemühen. (H. 4)

- Die meisten Artikel der drei näher untersuchten christlichen Jugendzeitschriften werden als Meinungsartikel verfaßt; dazu zählen Kommentar, Glosse, Rezension und Abhandlung. (H. 5)

- Die drei näher untersuchten christlichen Jugendzeitschriften bemühen sich um ein abwechslungsreiches, aufgelockertes Layout, indem sie unterschiedliche Schrifttypen, Farbe und verschiedenartige Illustrationen verwenden. Allerdings werden die gleichen Gestaltungselemente bei „Bravo" sehr viel häufiger verwendet und vielseitiger kombiniert. (H. 6)

- Die Themenkomplexe Kultur und Individueller Lebensbereich nehmen den größten Anteil der angebotenen Themenpalette ein. Damit schlagen die drei untersuchten christlichen Jugendmagazine eine ähnliche Richtung wie das kommerzielle Jugendmagazin „Bravo" ein. Allerdings ist der Anteil an Themen aus den Bereichen Kultur und Individueller Lebensbereich in "Bravo" noch ausgeprägter. $(\mathrm{H}$. 7)

- Innerhalb des Themenkomplexes Individueller Lebensbereich sind die Einzelthemen bei den christlichen Jugendmagazinen anders gewichtet als bei "Bravo“. Während "Bravo" den Schwerpunkt eindeutig auf das Thema "Sexualität" legt, taucht der sexuelle Aspekt - beispielsweise bei der Thematisierung von Partnerschaft in den christlichen Jugendmagazinen selten oder gar nicht auf. (H. 8)

- Explizit christliche bzw. religiöse Themen tauchen in den drei näher untersuchten Jugendmagazinen unterschiedlicher Konfession nur vereinzelt auf. (H. 9) 
- Der Anteil der Beiträge zu allgemeinen Themen, bej denen ein christlicher Bezug hergestellt wurde, ist verhältnismäßig groß. $(H$. 10)

- Sowohl in den explizit christlichen Beiträgen als auch in den Beiträgen mit christlichem Bezug wird der christliche Aspekt am häufigsten über Glaubenserfahrungen bzw. Vor- und Leitbilder vermittelt. (H. 11)

- Kirchliche Amtsinhaber nehmen sowohl in den explizit christlichen Beiträgen als auch in den Beiträgen mit christlichem Bezug selten Stellung zu den jeweiligen Themen. (H. 12)

\section{Ergebnisse der Befragung}

\section{Bestandsaufnahme}

Bei den untersuchten Zeitschriften handelt es sich größtenteils um Publikationen mit relativ geringer Auflage. Acht der zwölf Blätter haben eine verkaufte Auflage unter 20.000 Exemplaren, vier davon sogar von unter 10.000 Exemplaren (Tabelle 1). ${ }^{24}$ "Aktion" als einzige Zeitschrift mit sechsstelliger Auflagenzahl (120.000) wird allerdings kostenlos verteilt. Nimmt man alle Auflagen der christlichen Jugendzeitschriften zusammen, so machen diese knapp ein Viertel der "Bravo“-Auflage aus, die bei 1,3 Millionen verkaufter Exemplare liegt. Von den zwölf untersuchten Zeitschriften enthalten sieben Werbeanzeigen. Nach der redaktionellen Grundkonzeption lassen sich zwei Typen unterscheiden: Magazine und Themenhefte (je sechs Titel).

Personell sind die Redaktionen nur schwach besetzt: Keine der Jugendzeitschriften hat mehr als drei festangestellte Redakteure; die Hälfte verfügt nur über eine einzige Redakteurstelle. Alle greifen zusätzlich auf freie Mitarbeiter zurück. Die christlichen Jugendzeitschriften werden fast ausschließlich im Abonnement vertrieben; keine ist am Kiosk erhältlich. Dies hat schon Louis Bosshart bei den von ihm untersuchten katholischen Jugendzeitschriften in der Schweiz kritisiert. Dadurch bestünde die Gefahr des Abdriftens ins "Presseghetto". ${ }^{25}$

Die Zielgruppe sind junge Leute im Alter von 12 bis 40 Jahren. Die reinen Teenager-Zeitschriften richten sich an jüngere Jugendliche zwischen 12 und 18. Dazu zählen auf katholischer Seite "Junge Zeit", „Komm mit", "Provo", "Aktion", auf evangelischer Seite "Baustein"

24 Die Angaben hier und im folgenden beziehen sich auf den Zeitpunkt der schriftlichen Befragung (Sommer 1994). Spätere Änderungen sind nicht berücksichtigt (so erscheint etwa die "Junge Zeit" seit April 1995 unter dem Titel "X-mag").

25 Louis Bosshart, Christliche Presse im säkularen Umfeld, in: Communicatio Socialis 22 (1989) H. 1-2, 46-60, hier 49. 


\begin{tabular}{|c|c|c|c|c|c|c|}
\hline Titel & $\begin{array}{c}\text { Hintergiund } \\
\text { (kirchlich } \\
\text { konfessionell) }\end{array}$ & Träger & $\underset{j \text { jaündurngs- }}{\text { Gor }}$ & $\begin{array}{l}\text { verkaufte } \\
\text { Auflage }\end{array}$ & $\begin{array}{l}\text { Ausgaben } \\
\text { pro Jahr }\end{array}$ & $\begin{array}{r}\text { lerbreitu' } \\
\text { gebiere }\end{array}$ \\
\hline Junge Zeit & katholisch & \begin{tabular}{|l|} 
Deutsche \\
Bischofskonferenz
\end{tabular} & $1972^{\mathrm{b})}$ & 44000 & 12 & $\mathrm{D}$ \\
\hline Kontraste & katholisch & $\begin{array}{l}\text { Verlag Herder, } \\
\text { Freiburg }\end{array}$ & 1969 & 15000 & 4 & $\mathrm{D}, \mathrm{A}, \mathrm{Ct}$ \\
\hline 17 & katholisch & $\begin{array}{l}\text { Steyler Missionare, } \\
\text { Nettetal } \\
\end{array}$ & 1973 & 17200 & 11 & $\mathrm{D}, \mathrm{A}, \mathrm{CH}$ \\
\hline Provo & katholisch & $\begin{array}{l}\text { Publik-Forum Ver- } \\
\text { lagsges., Oberursel }\end{array}$ & 1992 & 32000 & 4 & $\mathrm{D}, \mathrm{A}, \mathrm{Cl}$ \\
\hline Komm mit & katholisch & $\begin{array}{l}\text { Komm-mit-Verlag } \\
\text { G. Stiff, Münster }\end{array}$ & 1974 & 6000 & 12 & $\mathrm{D}$ \\
\hline Aktion & katholisch & \begin{tabular}{|l|} 
Initiative Jugend $\&$ \\
Beruf, München \\
\end{tabular} & 1991 & 120000 & 2 & $\vec{D}$ \\
\hline Die Glocke & evangelisch & \begin{tabular}{|l|} 
Chr. Jugenddorfwerk \\
Deutschlands
\end{tabular} & 1948 & 26000 & 12 & $\mathrm{D}$ \\
\hline Baustein & evangelisch & $\begin{array}{l}\text { CVJM-Westbund, } \\
\text { W'uppertal } \\
\end{array}$ & 1948 & 8500 & 12 & $\mathrm{D}$ \\
\hline Dran & evangelisch & \begin{tabular}{|l}
$\begin{array}{l}\text { Bundes-Verlag, } \\
\text { Witten }\end{array}$ \\
\end{tabular} & $1993^{c)}$ & 15500 & 9 & $\mathrm{D}, \mathrm{A}, \mathrm{Cl}$ \\
\hline Contrapunkt & evangelisch & $\begin{array}{l}\text { Arbeitsgemeinschaft } \\
\text { MBK, Bad Salzuflen }\end{array}$ & $1968^{d)}$ & 7000 & 6 & $\overline{D, A, C F}$ \\
\hline Exact & erangelisch & $\begin{array}{l}\text { Veriag Wort im Bild, } \\
\text { Hammersbach }\end{array}$ & $1994^{e)}$ & 6000 & 12 & $\overline{\mathrm{D}, \mathrm{A}, \mathrm{CF}}$ \\
\hline Champ & freikirchlich & $\begin{array}{l}\text { Freie chr. Jugendge- } \\
\text { meinsch., Llidenscheid }\end{array}$ & 1989 & 11000 & 4 & $\mathrm{D}, \mathrm{A}, \mathrm{CF}$ \\
\hline Bravo & (kommerziell) & $\begin{array}{l}\text { Heinrich Bauer Verlag, } \\
\text { Hamburg }\end{array}$ & 1958 & 1.3 Mio. & 52 & $\overline{D, A, C H}$ \\
\hline
\end{tabular}

\begin{tabular}{|c|c|c|c|c|c|}
\hline $\begin{array}{l}\text { 'ertriebs } \\
\text { art (über- } \\
\text { wiegend) }\end{array}$ & $\begin{array}{c}\text { Preis pro } \\
\text { Heft } \\
\text { in DM }\end{array}$ & $\begin{array}{c}\text { Werbe- } \\
\text { anzeigen }\end{array}$ & $\begin{array}{c}\text { Art der } \\
\text { Zeitschriff }\end{array}$ & $\begin{array}{c}\text { Redakeure } \\
\text { (jest } \\
\text { angestellf) }\end{array}$ & $\begin{array}{c}\text { Zielgruppe } \\
\text { (Alter) }\end{array}$ \\
\hline Abo. & 3,- & ja & Magazin & $2-3$ & $13-18$ \\
\hline Abo. & 6,- & ja & Themenheft & $2-3$ & $14-40$ \\
\hline Abo. & 2,- & nein & Themenheft & I & $15-26$ \\
\hline Abo. & 4,- & nein & Magazin & 1 & $13-19$ \\
\hline Abo. & 1,50 & nein & Magazin & $2 \cdot 3$ & $12-18$ \\
\hline Abo. & kostenlos & nein & Themenheft & $2 \cdot 3$ & $15-19$ \\
\hline Abo. & 4,50 & ja & Magazin & $2 \cdot 3$ & $17-35$ \\
\hline Abo. & 1,60 & nein & Themenheft & 1 & $13-17$ \\
\hline Abo. & 5,80 & ja & Themenheft & I & $17-30$ \\
\hline Abo. & 4,- & ja & Themenhefi & 1 & $17-25$ \\
\hline Abo. & 3,67 & ja & Magazin & 1 & $16-35$ \\
\hline Abo. & 5,50 & ja & Magazin & 2.3 & $12-19$ \\
\hline Kiosk & 2,20 & ja & Magazin & über 10 & $13-17$ \\
\hline
\end{tabular}


und das freikirchliche Magazin "Champ". An die älteren Jugendlichen bzw. jungen Erwachsenen richten sich die katholischen Zeitschriften "Kontraste" und "17" sowie die evangelischen Blätter "Contrapunkt", "Die Glocke”, "Dran“ und „Exact". Bei der näheren Beschreibung ihres Zielpublikums wird deutlich, daß die meisten Chefredakteure trotz prinzipieller Offenheit für alle Jugendlichen in erster Linie junge, engagierte Christen ansprechen wollen. ${ }^{26}$

\section{Christliche Inhalte und Orientierung an den Jugendlichen}

Erwartungsgemäß bestätigten die Chefredakteure der untersuchten Blätter in der schriftlichen Befragung, daß sie mit ihrer Zeitschrift eine christliche Intention verfolgen. Die Vermittlung entsprechender Inhalte spielt in den meisten publizistischen Konzeptionen eine wichtige Rolle, womit die gleichlautende Hypothese (H. 1) als bestätigt gelten kann. Im Vordergrund steht dabei zunächst die Vermittlung von christlichen Grundwerten. Dann folgen die Einordnung der behandelten Themen in einen christlichen Gesamtzusammenhang sowie Informationen über aktuelle Ereignisse im christlichen/kirchlichen Bereich (Abbildung 1).

Abbildung 1: Konzeptionelle Berücksichtigung christlicher und nicht-christlicher Inhalte

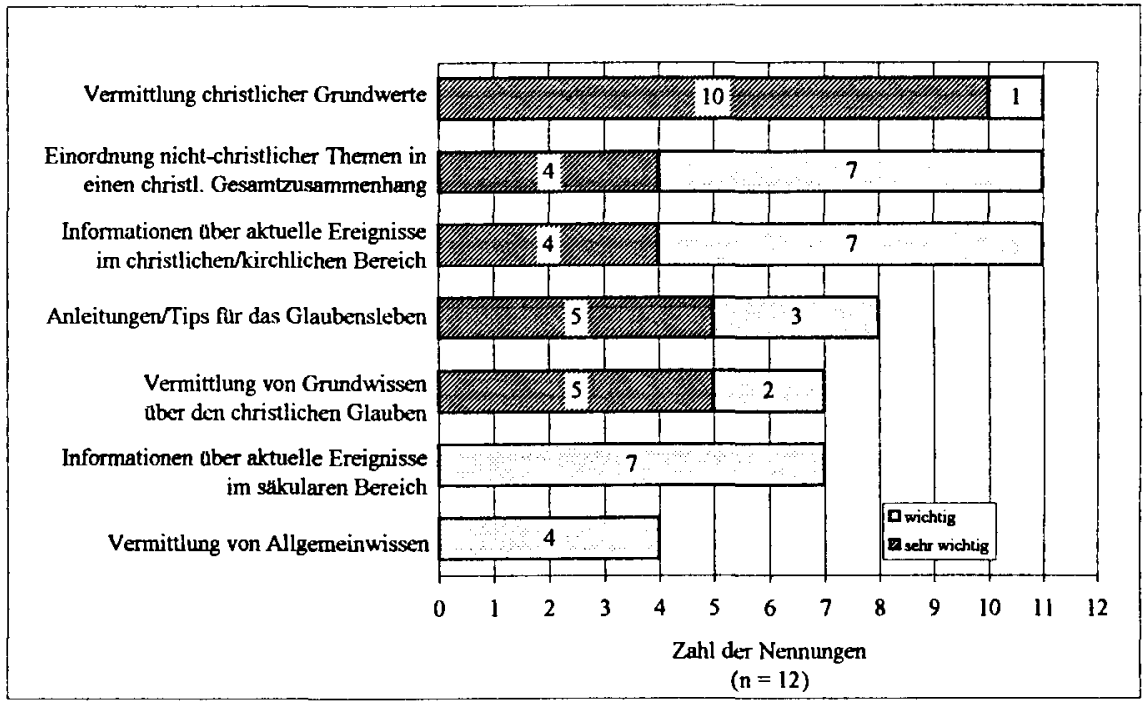

Die Hypothese, daß sich die Chefredakteure an den Jugendlichen orientieren und dies im Blatt entsprechend umsetzen wollen ( $H .2)$,

26 Nur neun der zwölf Chefredakteure haben sich zu dieser offenen Frage geäußert. Sieben davon beschrieben ihre Zielgruppe als junge, engagierte Christen. 
kann ebenfalls bestätigt werden. Bis auf eine Ausnahme halten es alle Chefredakteure für "wichtig" bis „sehr wichtig”, daß die jungen Leser die Zeitschrift als „ihre" Zeitschrift erleben, in der alles, was sie betrifft und interessiert, zur Sprache kommt (Abbildung 2). Wenn es darum geht, die Interessen der jungen Leser herauszufinden, wenden sich fast alle Chefredakteure direkt an solche Jugendliche, zu denen sie auch häufig persönlich Kontakt haben. In allen untersuchten Zeitschriften werden Jugendliche aktiv an der Heftgestaltung beteiligt.

\section{Abbildung 2: Konzeptionelle Orientierung an den Interessen Jugendlicher}

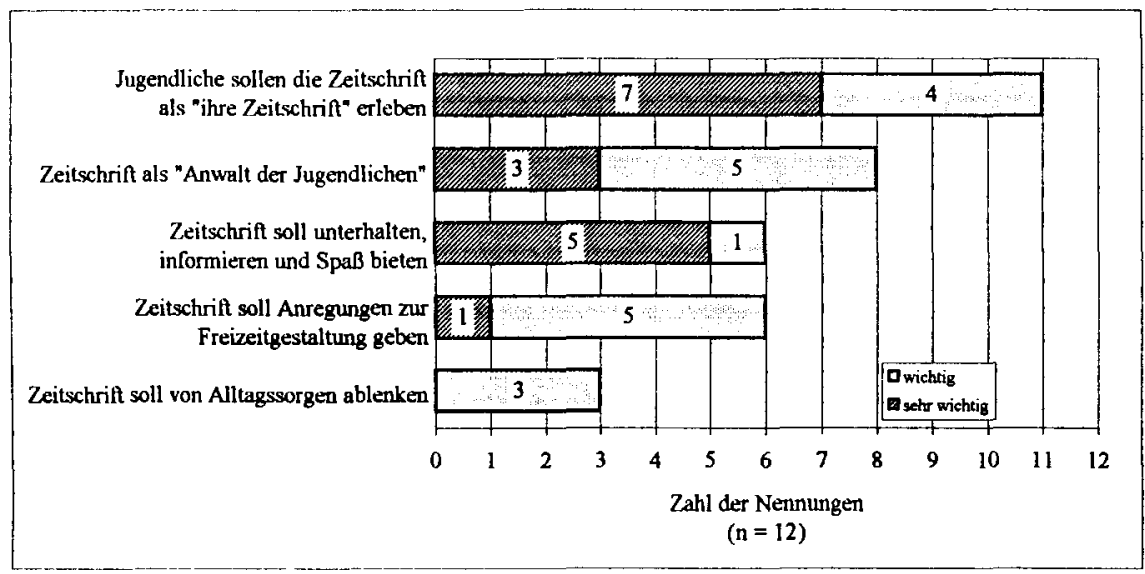

\section{Ergebnisse der Inhaltsanalyse und der Leitfadengespräche}

Inwieweit die christlichen Zeitschriften ihre publizistischen Konzeptionen umsetzen und sich tatsächlich an den Jugendlichen orientieren oder die als wichtig erachteten Inhalte in den Heften berücksichtigen, wurde exemplarisch bei den Magazinen "Junge Zeit" (katholisch), "Glocke" (evangelisch) und "Champ" (freikirchlich) im Vergleich zu „Bravo“ überprüft. Der Themenanalyse ging zunächst die Untersuchung der äußeren Erscheinung der christlichen Blätter im Vergleich zu "Bravo" voraus.

\section{Formale Kategorien}

\section{Darstellungsart}

Ein grundlegendes formales Merkmal zur Untersuchung der Zeitschriftenartikel waren zunächst die verschiedenen „Darstellungsarten".27 Alle redaktionellen Textbeiträge und eigenständigen Bildbeiträge

27 Bei „Bravo" wurden insgesamt 685 Artikel ermittelt, bei der “Jungen Zeit” 565; die vier Ausgaben von "Champ" enthielten 359 und die Hefte der „Glocke" 121 Artikel. 
der analysierten Zeitschriften wurden hier den einzelnen Kategorien zugeordnet (Tabelle 2). Dabei konnte festgestellt werden, daß sowohl in den christlichen. Magazinen als auch in "Bravo" Sachbeiträge und Medientips die häufigsten Darstellungsarten waren. In allen Zeitschriften lassen sich zwischen 41 und $62 \%$ der Artikel den beiden Kategorien zuordnen. Es handelt sich bei dieser Einordnung jedoch zunächst nur um eine formale, nicht um eine inhaltliche Abgrenzung. ${ }^{28}$ Die weiteren Darstellungsarten haben bei allen Zeitschriften einen Anteil von nur $17 \%$ oder weniger.

Als einziges christliches Magazin bietet die "Junge Zeit" in jeder Ausgabe eine Problemseite, auf der persönliche Fragen der Leser beantwortet werden. Die Artikel unter dieser Rubrik machen 3\% des Gesamtinhalts aus. In den beiden anderen christlichen Magazinen taucht diese Form der Lebenshilfe nur sporadisch auf. ${ }^{29}$ Die Artikel auf den Problemseiten von "Bravo" dagegen haben - gemessen an der Gesamtzahl der erfaßten Artikel - einen Anteil von 6\%.

Nur selten findet man in den Zeitschriften literarische Darstellungsarten im weitesten Sinn wie Gedichte und Liedtexte, Kurzgeschichten und Gebete. Sie machen in allen Magazinen einen Anteil von unter $6 \%$ aus. Dabei überrascht besonders, daß in den christlichen Jugendzeitschriften kaum Gebete oder besinnliche Texte zu finden sind. Unter den 565 erfaßten Artikeln der "Jungen Zeit" sind gerade zwei Gebete, bei "Champ" sind es ebenfalls nur zwei, bei der "Glocke" ist es sogar nur eines.

\section{Layout}

Die drei Chefredakteure der näher untersuchten christlichen Magazine sind sich laut ihren Aussagen in den Leitfadengesprächen bewußt, welche zentrale Rolle ein professionelles Layout spielt, um bei den Jugendlichen anzukommen. Die Ergebnisse der Inhaltsanalyse zeigen, $\mathrm{da} \beta$ sich alle drei Blätter um eine abwechslungsreiche Aufmachung bemühen. Allerdings schneidet "Bravo" im großen und ganzen am besten ab, womit die gleichlautende Hypothese (H. 6) bestätigt werden kann. "Bravo" hat sowohl den größten Farbanteil, den größten Bildanteil und die meisten Illustrationen pro Artikel als auch die abwechslungsreichste Anordnung dieser Illustrationen. Insgesamt betrachtet kommen die katholische "Junge Zeit" und das freikirchliche Magazin "Champ" der kommerziellen Konkurrenz noch am nächsten. Dagegen wirken sich bei der "Glocke" vor allem der hohe Schwarzweiß-

28 Der Begriff des Sachbeitrags ist sehr weit gefaßt und kann durchaus auch aus Meinungsdarstellungen bestehen; „Sachbeitrag“ (als Darstellungsart) ist also nicht gleichbedeutend mit "Information/Bericht“ (als Darstellungsform).

29 Dieses Ergebnis bezieht sich wie die anderen Resultate der Inhaltsanalyse auf die untersuchten Hefte des Jahrgangs 1993. Seit der Blattreform Anfang 1994 hat auch die "Glocke" eine feste Beratungsseite mit dem Titel „Mein Problem". 
Tabelle 2: Darstellungsarten im Vergleich

\begin{tabular}{|l|c|c|c|c|c|c|c|c|}
\hline \multirow{2}{*}{ Darstellungsart } & \multicolumn{2}{c|}{ Junge Zeit } & Die Glocke & \multicolumn{2}{c|}{ Champ } & \multicolumn{2}{c|}{ Bravo } \\
\cline { 2 - 8 } & Anzahl & Prozent & Anzahl & Prozent & Anzahl & Prozent & Anzahl & Prozent \\
\hline Sachbeitrag & 119 & 21,1 & 43 & 35,5 & 110 & 30,6 & 174 & 25,4 \\
Medientip & 130 & 23,0 & 32 & 26,4 & 58 & 16,2 & 107 & 15,6 \\
Freizeittip & 54 & 9,6 & 14 & 11,6 & 18 & 5,0 & 69 & 10,1 \\
Problemseite & 16 & 2,8 & - & - & - & - & 44 & 6,4 \\
Leserbrief & 26 & 4,6 & 5 & 4,1 & 35 & 9,7 & 18 & 2,6 \\
Such-/Kontaktanzeigen & 91 & 16,1 & - & - & 61 & 17,0 & 76 & 11,1 \\
Poster & 9 & 1,6 & - & - & 8 & 2,2 & 19 & 2,8 \\
Einzelbild & 7 & 1,2 & - & - & - & - & - & - \\
Witz/Cartoon & 36 & 6,4 & 2 & 1,7 & 21 & 5,9 & 90 & 13,1 \\
RătseV/Quiz & 21 & 3,7 & 7 & 5,8 & 21 & 5,9 & 25 & 3,7 \\
TV-Programm/Hitliste & 2 & 0,4 & - & - & - & - & 32 & 4,7 \\
Gebet & 2 & 0,4 & 1 & 0,8 & 2 & 0,6 & - & - \\
Gedicht/Liedtext & 28 & 5,0 & - & - & 13 & 3,6 & 9 & 1,3 \\
Kurzgeschichte & 4 & 0,7 & 4 & 3,3 & - & - & 8 & 1,2 \\
Editorial & 3 & 0,5 & 3 & 2,5 & 2 & 0,6 & 4 & 0,6 \\
Vorschau/Impressum & 13 & 2,3 & 6 & 5,0 & 6 & 1,7 & 6 & 0,9 \\
\hline Artikel gesamt: & 565 & 100 & 121 & 100 & 359 & 100 & 685 & 100 \\
\hline
\end{tabular}

Anteil (58\%) und die Textlastigkeit (der Bildanteil beträgt nur ein Drittel) negativ auf das Layout aus. ${ }^{30}$

\section{Länge}

Des weiteren kann den christlichen Jugendzeitschriften eine Orientierung am heutigen Kommunikationsstil der jungen Leute bescheinigt werden. So bestätigt sich für alle drei Blätter die Hypothese, daß die Artikel überwiegend sehr kurz sind, d.h. kürzer als eine halbe Seite $(H$. 3). Im Vergleich dazu hat "Bravo" die meisten sehr kurzen Beiträge mit einem Anteil von $83 \%$ aller Artikel, jedoch dicht gefolgt von der "Jungen Zeit" mit $82 \%$ und "Champ" mit 74\%. Dagegen enthielten die untersuchten Ausgaben der "Glocke” lediglich 53\% sehr kurze Artikel.

30 Beides hat sich seit der Reform zu Beginn des Jahres 1994 offenbar etwas verbessert. 


\section{Sprachstil}

Auch sprachlich orientieren sich die christlichen Jugendzeitschriften an ihrer Zielgruppe, indem sie ohne Ausnahme in etwa 80 bis $90 \%$ der Artikel den Jargon der Jugendlichen verwenden - allerdings in unterschiedlicher Intensität. Insgesamt kann die entsprechende Hypothese (H. 4) bestätigt werden. Auffallend ist, daß das freikirchliche Magazin "Champ" am häufigsten und ausgeprägtesten jugendliche Trend- und Modewörter benutzt und damit sogar noch vor "Bravo" liegt. Am meisten Zurückhaltung wurde wiederum bei der "Glocke" festgestellt, die sich jedoch auch in diesem Punkt durch die Reform verstärkt auf die Jugendlichen zubewegen will.

\section{Darstellungsform}

Die Hypothese hinsichtlich der Darstellungsform (H. 5) hat sich ebenfalls bestätigt: Ähnlich wie bei „Bravo“ überwiegen bei allen drei christlichen Magazinen Meinungsartikel wie Rezensionen, Kommentare, Glossen und Abhandlungen. Dabei handelt es sich jedoch meist um kurze (redaktionelle und Leser-)Beiträge, bei den längeren Artikeln lassen sich kaum noch Meinungsäußerungen finden. Eine Ausnahme stellt auch hier wieder die "Glocke" dar, wobei dies laut Auskunft der Chefredaktion mit der Blattreform geändert wurde. Die Redaktionsleiter der "Glocke" und der "Jungen Zeit" betonten in den Leitfadengesprächen, daß sie sich bewußt mit Meinung zurückhalten und den Jugendlichen vielmehr Hintergrundinformation zur eigenen Meinungsbildung an die Hand geben wollen. Auch halten es alle drei Chefredakteure für wichtig, daß die Jugendlichen selbst im Blatt zu Wort kommen und ihre Meinung äußern. Dies geschieht bei den drei christlichen Magazinen im Durchschnitt in jedem vierten Artikel. In der "Jungen Zeit" und in "Champ" handelt es sich hauptsächlich um kurze Leserbeiträge, während in der "Glocke" die Äußerungen Jugendlicher innerhalb von redaktionellen Texten überwiegen. Bei "Bravo" kommen Jugendliche sogar in jedem dritten Artikel zu Wort, allerdings fast ausschließlich in Form von extrem kurzen Leserbeiträgen und -anfragen.

\section{Themenspektrum}

Ein weiteres zentrales Untersuchungsfeld war die Themenanalyse. Hier sind sich die Chefredakteure der drei christlichen Blätter einig, daß eine für Jugendliche attraktive christliche Zeitschrift ein breites Spektrum bieten sollte. Die Umsetzung dieser Vorstellung gelingt der "Jungen Zeit" und der "Glocke" am besten. In beiden Blättern werden neben christlichen Themen auch Themen aus den Bereichen Kultur, Individueller Lebensbereich, Gesellschaft und Sport aufgegriffen (Abbildung 3). Für "Champ" ergibt sich im Vergleich dazu ein etwas eingeschränktes Themenspektrum, da hier die Gesellschaftsrelevanten Themen und auch der Individuelle Lebensbereich nur sehr marginal behandelt werden. Nach Aussage des Chefredakteurs ist dies jedoch eigentlich nicht beabsichtigt; vielmehr sei die Personalknappheit in der Redaktion dafür verantwortlich. 


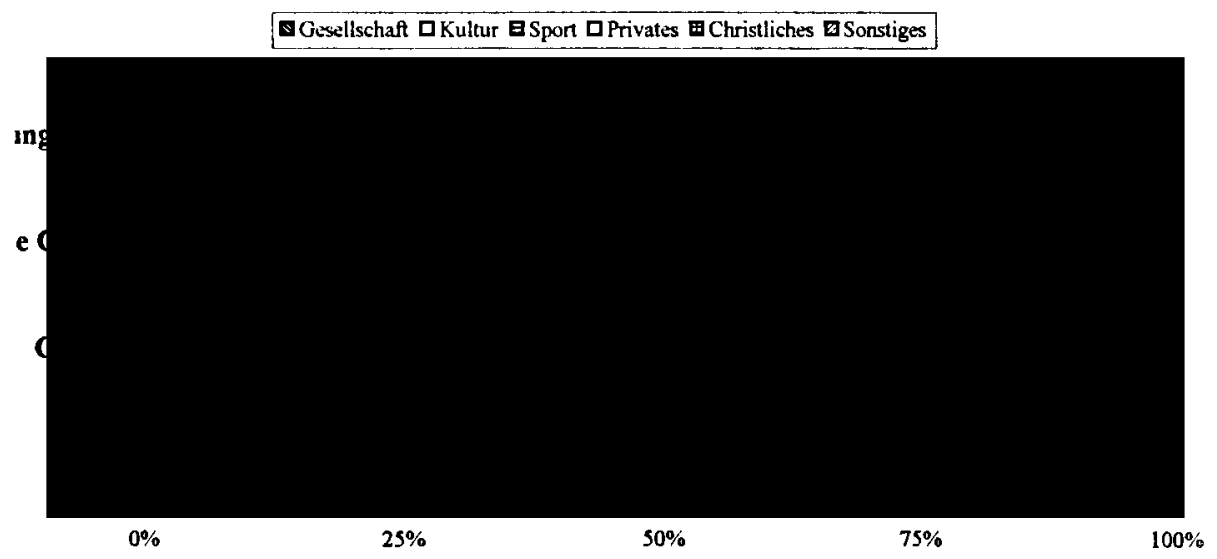

\section{Kulturelle Themen}

Übereinstimmend nehmen bei allen drei christlichen Blättern die kulturellen Themen ${ }^{31}$ den größten Anteil am Gesamtinhalt ein (,Junge Zeit": 43\%, "Glocke": 38\%, "Champ": 49\%). Die Hypothese, die von einer entsprechenden thematischen Schwerpunktsetzung ausgeht $(H .7)$, kann auch in der Hinsicht als bestätigt gelten, daß popularkulturelle Themen in "Bravo" noch weit stärker vertreten sind (70\%). Während jedoch die "Junge Zeit" und die "Glocke" in den Kulturbeiträgen vorwiegend säkulare Musik, Film/Fernsehen und Literatur thematisieren, beschränkt sich die Kulturberichterstattung in "Champ" fast ausschließlich auf die christliche Musikszene. In bewußter Abgrenzung zu „Bravo“ wollen alle christlichen Magazine zudem auf einen Starkult verzichten.

\section{Individueller Lebensbereich: Partnerschaft und Sexualität}

Der zweite Teil dieser Hypothese, wonach neben den Kulturthemen der Individuelle Lebensbereich sowohl bei den christlichen Zeitschriften als auch bei „Bravo" einen weiteren thematischen Schwerpunkt bildet, kann nur für die "Glocke" und die "Junge Zeit" bestätigt werden (Abbildung 4). Dabei ist der Anteil der Artikel zum Individuellen Lebensbereich in der "Jungen Zeit" sogar noch höher als in "Bravo". Innerhalb dieses Themenfelds tauchen die Einzelthemen Partnerschaft und Sexualität neben Freizeit/Hobby/Trends - sowohl in "Bravo" als auch in der "Glocke" und in der "Jungen Zeit" am häufigsten auf. Auffällig ist, daß bei den christlichen Magazinen und "Bravo" gleichermaßen Themenbereiche aus dem direkten Umfeld der Jugendlichen wie Familie/Eltern oder auch Erwachsenwerden/Pubertät äußerst selten angesprochen

31 Kultur wird hier im weitesten Sinne verstanden. Im Hinblick auf Jugendliche meint dies vor allem Popkultur und Showbusineß, aber auch Jugendliteratur. 
wurden. Lediglich der Bereich Schule/Beruf wird - im Gegensatz zu "Bravo" - in der "Jungen Zeit" und in der "Glocke" stärker thematisiert.

\section{Abbildung 4: Einzelthemen in indiviluellen Lebensbereich}

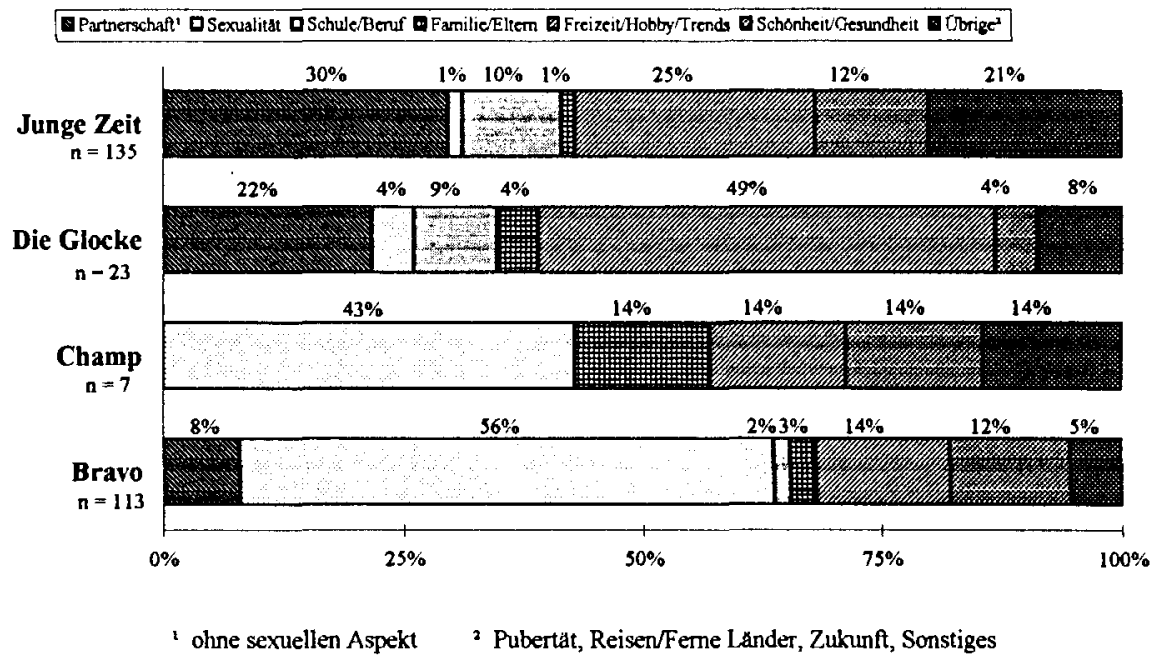

Bei näherer Betrachtung clei Artikel zu Partnerschaft und Sexualität werden bezüglich der inhaltlichen Aufarbeitung große Unterschiede zwischen "Bravo" und den christlichen Magazinen deutlich. Es bestätigt sich die Hypothese, daß bei "Bravo" die Beziehungsthemen weitgehend auf den sexuellen Aspekt reduziert sind, während die christlichen Magazine den sexuellen Bereich bei der Thematisierung von Partnerschaft tabuisieren (H. 8). In den untersuchten Ausgaben fiel auf, daß Sexualität - wenn überhaupt - in einem anderen Kontext angesprochen wurde, beispielsweise in Berichten über sexuellen Mißbrauch bei Kindern.

Für diese weitgehende Ausklammerung der Sexualität nannten die Chefredakteure ganz unterschiedliche Gründe. Während man bei "Champ" Geld- und Personalmangel als äußere Zwänge anführte und gleichzeitig prinzipielle Offenheit signalisierte, das Thema aufzuarbeiten - allerdings aus streng biblischer Sicht -, sprach der Chefredakteur der katholischen "Jungen Zeit" von "internen Schwierigkeiten" während des Untersuchungszeitraums mit der Deutschen Bischofskonferenz als Herausgeberin des Blattes. Bei der "Glocke" wurde inzwischen - im Rahmen der Reform Anfang 1994 - die prinzipielle Zurückhaltung gegenüber der Thematisierung von Sexualität aufgegeben und eine feste Problemseite eingerichtet, die laut Aussagen der Redationsleitung bei entsprechenden Anfragen auch das Thema Sex und Partnerschaft behandelt. 
Gesellschaftsrelevante Themen

Allgemeine gesellschaftliche Themen stehen bei allen untersuchten Magazinen an nachgeordneter Stelle, werden jedoch zumindest bei der "Glocke" und der "Jungen Zeit" mit einem Anteil von $20 \mathrm{bzw}$. 12\% am Gesamtinhalt noch relativ häufig thematisiert. Im Vordergrund stehen dabei die Berichtsfelder Politik, Wirtschaft und Soziales, gefolgt von Beiträgen über Umweltthemen. Von "Champ“ und "Bravo" werden diese Themen stark vernachlässigt.

\section{Christliche Inhalte}

Bei der Aufarbeitung bzw. Gewichtung der christlichen Inhalte treten große Unterschiede zwischen den drei Zeitschriften auf, die vermutlich auf den kirchlichen Hintergrund zurückzuführen sind. Beim freikirchlichen Magazin "Champ" ist die stärkste Betonung christlicher Inhalte bzw. ein ausgeprägter und offensichtlicher christlicher Bezug bei nahezu jedem Thema feststellbar. Die Vermittlung der christlichen Botschaft sei es in expliziter Form oder indem in Artikeln zu anderen Themen ein christlicher Bezug hergestellt wird - nimmt in den untersuchten Ausgaben trotz betont lockerer Sprache missionarische Züge an, wie sie dem freikirchlichen Bereich generell eigen sind. Bei der "Jungen Zeit" und der "Glocke" dagegen, die den beiden Volkskirchen nahestehen, liegt der Anteil der Artikel mit christlichen Inhalten lediglich bei 14 bzw. 30\%. Innerhalb der beiden großen Konfessionen macht sich seit längerem unter den Jugendlichen eine skeptische, ablehnende Haltung gegenüber der Kirche bemerkbar. Von daher kann man vermuten, daß die "Junge Zeit" und die "Glocke" bewußt auf sehr behutsame Art und Weise versuchen, christliche Inhalte zu vermitteln.

Abbildung 5: Artikel mit christlichen Inhalten

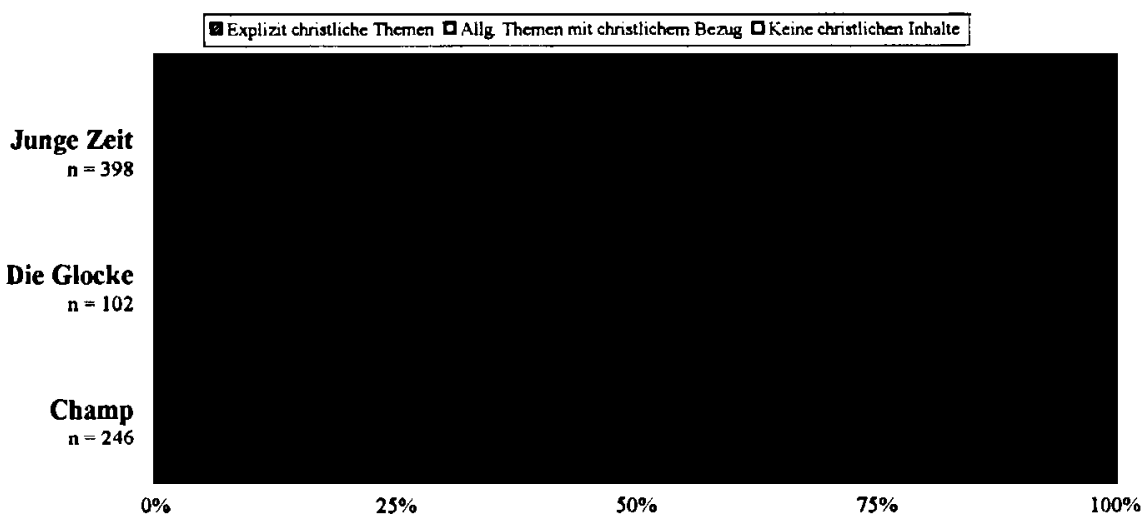




\section{Explizit christliche Beiträge}

Betrachtet man die explizit christlichen Beiträge für sich allein, so bestätigt sich für die "Junge Zeit" und die "Glocke" die Hypothese $(\mathrm{H}$. 9), wonach diese Art der christlichen Inhalte nur vereinzelt auftauchen (5 bzw. 16\% Anteil). Dagegen nehmen die explizit christlichen Beiträge bei "Champ" einen Anteil von 28\% ein. Ein christlicher Bezug wird darüber hinaus in $61 \%$ der Artikel hergestellt, womit nur für "Champ" die Hypothese bestätigt werden kann, daß der Anteil der Beiträge mit einem solchen Bezug verhältnismäßig groß ist (H. 10).

Diese Befunde unterstützen den Eindruck, daß „Champ“ seine christliche Intention sehr direkt und missionarisch umsetzt - anders als die "Junge Zeit" und die "Glocke", wo neben den vereinzelten explizit christlichen Beiträgen nur selten Beiträge mit christlichem Bezug auftauchen (9 bzw. 14\%). Darüber hinaus ist der christliche Bezug in den entsprechenden Artikeln überwiegend nur schwach ausgeprägt. Einschränkend muß angemerkt werden, daß im Rahmen der durchgeführten quantitativen Inhaltsanalyse christliche Bezüge „zwischen den Zeilen" nicht erfaßt werden konnten, obwohl die Chefredakteure dieser beiden Zeitschriften nach eigener Aussage genau darauf setzen. Da jedoch ein christlicher Bezug "zwischen den Zeilen“ naturgemäß nur sehr schwach ausgeprägt sein kann, ist nicht davon auszugehen, daß dadurch der dargestellte Befund - Zurückhaltung bei christlichen Inhalten - verfälscht wird.

\section{Themen der christlichen Beiträge}

Hinsichtlich des Inhalts der (explizit und implizit) christlichen Beiträge kann die entsprechende Hypothese (H. 11) - mit Einschränkungen - für alle drei Magazine bestätigt werden. Das Themenfeld Glaubenserfahrungen/Christliche Vorbilder, das laut Hypothese am häufigsten auftauchen soll, spielt bei den drei christlichen Blättern tatsächlich eine zentrale Rolle, liegt jedoch nur bei der "Jungen Zeit" ganz vorn, während es bei der "Glocke" und "Champ" den zweiten Platz belegt. Allerdings werden bei den beiden Zeitschriften mit kirchlich-konfessionellem Hintergrund die Glaubenserfahrungen nur am Rande behandelt (d.h. der christliche Bezug ist schwach ausgeprägt), während sie im freikirchlichen "Champ" im Mittelpunkt stehen.

\section{Kirchliche Amtsinhaber}

Übereinstimmend für alle drei Magazine konnte festgestellt werden, daß kirchliche Amtsinhaber in den christlichen Beiträgen nur selten als Autoren oder in Form von Zitaten zu Wort kommen: bei der "Jungen Zeit" in $16 \%$, bei der "Glocke" in $10 \%$ und bei "Champ" in $6 \%$ der einschlägigen Artikel. Die diesbezügliche Hypothese (H. 12) wird damit voll bestätigt. Während das freikirchliche Magazin "Champ" nach eigenen Angaben aus stilistischen Gründen auf Zitate kirchlicher Funktionäre verzichtet, führen die Chefredakteure der Blätter mit kirchlich-traditionellem Hintergrund, "Junge Zeit" und "Glocke", auch 
hier die zunehmende Skepsis der Jugendlichen gegenüber der Kirche und den kirchlichen Amtsinhabern als Grund an.

\section{Resümee}

Alle Chefredakteure der untersuchten Jugendzeitschriften verfolgen eine christliche Zielsetzung, die aber - wie die exemplarische Analyse von drei Blättern zeigte - auf ganz unterschiedliche Weise umgesetzt wird. Die Unterschiede in der Häufigkeit und Gewichtung christlicher Inhalte deuten darauf hin, daß die "Macher" dieser drei Zeitschriften unterschiedlich darüber denken, wie man Jugendliche am besten mit solchen Werten und Themen erreichen kann. Zwar sind sich die Redaktionsleiter darin einig, daß sie sich mit ihren Zeitschriften an den (Medien)Gewohnheiten und Interessen der Jugendlichen orientieren müssen. Dies setzt jedoch ein detailliertes Wissen über die Zielgruppen voraus. Wer sind die Leser der Zeitschriften? Für welche Themen interessieren sie sich, und welche Rolle spielen der christliche Glaube und die Kirche in ihrem Leben? Wie beurteilen die Jugendlichen selbst die Zeitschriften?

Offensichtlich schätzen die Chefredakteure ihre Zielgruppe und deren Wünsche oder Interessen unterschiedlich ein. Dies liegt möglicherweise darin begründet, daß ihnen (noch) keine gesicherten Informationen über die Leser ihrer Zeitschriften vorliegen. Eine andere Möglichkeit ist, daß sich die drei Zeitschriften doch nicht an alle Jugendlichen richten, sondern ihre Zielgruppe enger fassen. Diesen Schluß legt beispielsweise die stark missionarische Aufarbeitung christlicher Themen im freikirchlichen Magazin "Champ" nahe, das sich in erster Linie an Jugendliche richtet, die entsprechend engagiert oder zumindest christlichen Inhalten gegenüber sehr aufgeschlossen sind.

Über die Leserschaft christlicher Jugendzeitschriften liegen bislang keine umfassenden empirischen Studien vor, an denen sich die Chefredakteure orientieren könnten. Die wenigen bisher durchgeführten Untersuchungen beschränken sich entweder auf Teilbereiche, liegen bereits Jahrzehnte zurück oder wurden außerhalb Deutschlands durchgeführt. ${ }^{32}$ So umfaßt eine 1993 durchgeführte Akzeptanzanalyse bundesweiter katholischer Jugendzeitschriften fast ausschließlich Verbandsblätter. Zudem handelt es sich dabei um eine kircheninterne Untersuchung, die der Öffentlichkeit nicht zugänglich ist. Diese Studie ist aber ein möglicher Anknüpfungspunkt für weitere Untersuchungen, die dann konfessionsübergreifend sein sollten. Außerdem wäre es wichtig, dem schnellen „Generationswechsel" unter Jugendlichen stets durch neue Erhebungen Rechnung zu tragen.

32 Vgl. etwa Heinz Pürer, Kirchliche Jugendpublizistik in Österreich, in: Communicatio Socialis 7 (1974) H. 2, 137-151. 
Für die Jugendzeitschriften wäre es darüber hinaus von Vorteil, derartige Untersuchungen breit genug anzulegen, um über den bereits erschlossenen Leserkreis hinaus Informationen über Interessen und Glaubenshaltungen gerade derjenigen Jugendlichen $\mathrm{zu}$ gewinnen, die christliche Zeitschriften (noch) nicht lesen bzw. häufig nicht einmal von der Existenz dieser Blätter wissen. Solange die Zeitschriften ausschließlich im Abonnement vertrieben werden, dürften sich die Leser hauptsächlich aus dem kirchlichen Umfeld rekrutieren.

Um darüber hinaus Jugendliche anzusprechen, die nicht unbedingt in kirchlichen Kreisen verkehren, aber trotzdem Interesse an Glaubensfragen haben, wäre zusätzlich der Vertrieb über den Kiosk sinnvoll und notwendig. Nach Aussage der Chefredakteure ist diese Variante jedoch zu teuer. Als praktikable Alternative bliebe noch die Auslage in öffentlichen Bibliotheken, die von den befragten Chefredakteuren auch als gangbarer Weg eingeschätzt, erstaunlicherweise bisher jedoch noch nicht in die Tat umgesetzt wurde. Damit wäre ein erster Schritt in eine breitere Öffentlichkeit getan. ${ }^{33}$

Christliche Jugendzeitschriften bewegen sich auf einem schmalen Grad: Sie sollen sowohl dem gesellschaftlichen Dialog dienen als auch dem Verkündigungsanspruch der Kirchen gerecht werden. In einer säkularisierten Umwelt ist das alles andere als leicht - zumal die kommerziellen Kommunikationsangebote durch ihren Anpassungskurs weit weniger "Widerstände" für die Rezeption bereithalten.34 Die untersuchten Zeitschriften unternehmen viele Anstrengungen, um Jugendliche in ihrer Lebenswelt anzusprechen und ihnen ein Forum zu bieten. Von kirchenoffizieller Seite könnten sie dazu mehr Unterstützung gebrauchen.

SUMMARY: Between preaching and dialogue - Intentions and contents of nation-wide Christian Youth-magazines

More and more people - especially young ones - are leaving the Church and turn away from Christian faith. At the same time, there is an unbroken longing for religious and spiritual uplifting. Taking this into consideration, this article examines in which way the producers of nation-wide published German Youth-magazines try to attract their target readers.

The article includes a survey on 12 existing publications in general and goes into a deeper examination of three of them, combined with interrogations of the editors-in-chief.

33 "X-mag" (bis 1995 "Junge Zeit") hat inzwischen den weiterführenden Weg beschritten und ist seit kurzem - zunächst probeweise - in ausgewählten Buchhandlungen und Kiosken erhältlich (telefonische Auskunft der Redaktion am 11. Januar 1996).

34 Vgl. dazu neuerdings Andreas Vogel, Die Leserschaft der populären Jugendpresse. Markt- und Leseranalyse, in: Media Perspektiven 1996, H. 1, $18-29$. 
The examination proofs that Christian Youth-magazines have to move on knife's edge: They want to follow the actual dialogue as well as the claim of Church to proclaim faith. This is difficult in a totally secularized society, all the more as commercial secular Youth-magazines by their open and liberal contents offer less reasons for refusal to the readers.

RÉSUMÉ: Entre proclamation et dialogue. Intentions et contenus de revues chrétiennes pour la jeunesse qui dépassent le cadre régional

De plus en plus de gens - soutout les jeunes - se détournent de l'église et de la foi chrétienne. En même temps, il existe quand même un besoin ininterrompu de religiosité et de spiritualité. Face à cela, on cherche à savoir comment les producteurs de revues chrétiennes pour la jeunesse qui paraissent dans toute $l^{\prime}$ Allemagne, essaient d'atteindre leur groupe-cible. L'etude donne un aperçu de 12 de ces revues.

De plus, trois journaux choisis ont été minutieusement étudiés et l'on a posé des questions a leurs rédacteurs en chef. Il apparaît que les revues chrétiennes pour la jeunesse n'ont qu'un espace restreint: Elles doivent à la fois servir le dialogue dans la société et satisfaire au droit de proclamation des églises. Dans un monde séculaire, cela est bien moins que simple - d'autant plus que les offres de communications commerciales avec leur méthode d'adaptation présente beaucoup moins de "résistance" à la réception.

RESUMEN: Entre predicación y diálogo - Intenciónes y contenidos de las revistas juveniles cristianas de ámbito supraregional

Cada vez más personas - sobre todo jóvenes - se alejan de la iglesia y de la fé cristiana. Simultaneamente existe una necesidad inquebrantable de religiosidad y espiritualidad. En este contexto se examina la cuestión de cómo los editores de revistas juveniles cristianas, a lo largo y ancho del país, tratan de llegar a sus lectores.

El estudio nos da una visión de 12 revistas juveniles cristianas de ámbito supraregional. Tres de estas revistas fueron además examinadas y sus redactores jefes fueron consultados.

Se muestra que tales revistas se mueven en un campo muy estrecho: tienen que servir al diálogo social, al mismo tiempo que corresponder a las pretenciones de anunciación del evangelio por parte de las iglesias. En un ambiente secularizado, esta tarea es todo lo contrario de fácil - considerando que las ofertas comunicativas comerciales por su orientación de adaptación provocan mucha menor “resistencia” para su recepción. 